\title{
Patologia epatica in bambini e adolescenti con diabete mellito tipo 1: un legame tra controllo glicemico ed epatopatia
}

\author{
Antonio Siena ${ }^{1} \cdot$ Raffaella Buzzetti $^{1}$
}

Accettato: 18 dicembre 2020 / Pubblicato online: 18 maggio 2021

(c) The Author(s) 2021

\section{Commento a:}

Liver disease in children and adolescents with type 1 diabetes mellitus: a link between glycemic control and hepatopathy.

M.H. El-Sayed, R.A. Thabet, M.T. Hamza,

M.S. Hussein, M.M. El Saeed.

Diabetes Res Clin Pract (2020) 170:108458

I soggetti affetti da diabete mellito tipo 1 (DMT1) con scarso controllo glicemico possono sviluppare una particolare patologia epatica, nota come epatopatia glicogenica [1]. Tale patologia, reversibile se trattata precocemente, è dovuta all'accumulo di glicogeno negli epatociti ed è caratterizzata da epatomegalia e aumento delle transaminasi [2].

La nonalcoholic fatty liver disease (NAFLD), tipica dei soggetti con DMT2 e pluri-patologie metaboliche, è più rara nei soggetti con DMT1, specie se giovani, e non presenta la tipica reversibilità dell'epatopatia glicogenica dopo ottenimento di un buon controllo glicemico [3]. Questo studio di El-Sayed e collaboratori ha come obiettivo primario quello di valutare la presenza di patologia epatica in bambini e adolescenti con DMT1. L'obiettivo secondario è rappresentato dalla valutazione degli effetti che il miglioramento del controllo glicemico può avere sulla funzionalità epatica. Lo studio, di tipo trasversale, è stato condotto su 107 bambini e adolescenti (età inferiore ai 18 anni), normopeso, affetti da DMT1. Tutti i soggetti sono stati sottoposti a dosaggio di transaminasi (ALT e AST) e di HbA1c, e a ecografia addomino-pelvica; tutti i soggetti con transaminasi elevate

R. Buzzetti

raffaella.buzzetti@uniroma1.it

1 Dipartimento di Medicina Sperimentale, Sapienza Università di Roma, Roma, Italia (oltre 3 volte il limite superiore di riferimento) sono stati poi sottoposti a fibroscan. Novantotto di questi pazienti avevano transaminasi normali, mentre i restanti 9 avevano ALT e AST elevate. Il gruppo di pazienti con transaminasi normali aveva un valore medio di HbAlc di $8,2 \%$ e un solo paziente presentava epatomegalia all'ecografia; nel gruppo di pazienti con transaminasi elevate, l'HbA1c media era di $10,8 \%$ e tutti presentavano epatomegalia; il fibroscan effettuato in quest'ultimo gruppo ha mostrato un fibrosis score alterato in tutti i pazienti (valore medio $=1$ ).

Tutti i pazienti con transaminasi elevate sono stati inseriti in un programma di miglioramento del controllo glicemico, tramite modifiche riguardanti lo stile di vita e l'ottimizzazione della terapia insulinica.

Un adeguato controllo glicemico ha determinato dopo un anno una significativa riduzione nelle transaminasi (valori medi ALT $=25 \mathrm{U} / \mathrm{L}$ e AST $=29 \mathrm{U} / \mathrm{L}$ ), nel fibroscan fibrosis score (valore medio $=0$ ) e nell' HbA1c (valore medio $=9 \%), p=0,003, p=0,01$ e $p=0,003$, rispettivamente.

Concludendo, lo scompenso metabolico è un fattore predisponente a patologie epatiche in pazienti con diabete mellito. Soggetti giovani con DMT1 e scarso controllo della malattia possono presentare una particolare patologia epatica, nota come epatopatia glicogenica, che, se riscontrata in fase non fibrotica, può essere reversibile mediante miglioramenti comportamentali e terapeutici.

Funding Note Open access funding provided by Università degli Studi di Roma La Sapienza within the CRUI-CARE Agreement.

Nota della casa editrice Springer Nature rimane neutrale in riguardo alle rivendicazioni giurisdizionali nelle mappe pubblicate e nelle affiliazioni istituzionali. 
Open Access This article is licensed under a Creative Commons Attribution 4.0 International License, which permits use, sharing, adaptation, distribution and reproduction in any medium or format, as long as you give appropriate credit to the original author(s) and the source, provide a link to the Creative Commons licence, and indicate if changes were made. The images or other third party material in this article are included in the article's Creative Commons licence, unless indicated otherwise in a credit line to the material. If material is not included in the article's Creative Commons licence and your intended use is not permitted by statutory regulation or exceeds the permitted use, you will need to obtain permission directly from the copyright holder. To view a copy of this licence, visit http://creativecommons.org/licenses/by/4.0/.

\section{Bibliografia}

1. Parmar N, Atiq M, Austin L et al (2015) Glycogenic hepatopathy: thinking outside the box. Case Rep Gastroenterol 9(2):221-226

2. Khory J, Zohar Y, Shehadeh N et al (2018) Glycogenic hepatopathy. Hepatobiliary Pancreat Dis Int 17(2):113-118

3. Munns CF, McCrossin RB, Thomsett MJ, Batch J (2000) Hepatic glycogenosis: reversible hepatomegaly in type 1 diabetes. J Paediatr Child Health 36(5):449-452 